\title{
Cidades inteligentes e sustentáveis: análise e definições acerca da literatura
}

Este artigo tem como objetivo compreender os conceitos da literatura acerca das cidades inteligentes e sustentáveis. O estudo fornece uma visão abrangente dos conceitos que caracterizam as cidades inteligentes e sustentáveis na contemporaneidade. Para este estudo foram analisados 32 artigos científicos relacionados que versavam sobre práticas das cidades inteligentes e sustentáveis. Os resultados encontrados demonstram um agrupamento que destaca a, qualidade de vida infraestrutura, serviços, tecnologia de informação e comunicação - TIC, inteligência, cidadão, sociedade, meio ambiente, sustentabilidade, governança, gestão, economia, finanças e mobilidade. Percebeu-se uma forte ligação entre os TICs como elemento norteador para concepção das cidades inteligentes e sustentáveis.

Palavras-chave: Cidades inteligentes; Cidades sustentáveis; TICs; Revisão da literatura.

\section{Smart and sustainable cities: analysis and definitions about literature}

This article aims to understand the concepts in the literature about smart and sustainable cities. The study provides a comprehensive overview of the concepts that characterize smart and sustainable cities today. For this study, 32 related scientific articles were analyzed, dealing with smart and sustainable city practices. The results found demonstrate a grouping that highlights, quality of life, infrastructure, services, information and communication technology - TCl, intelligence, citizen, society, environment, sustainability, governance, management, economy, finance and mobility. A strong link between TCls was perceived as a guiding element for the design of smart and sustainable cities.

Keywords: Smart cities; Sustainable cities; ICTs; Literature review.

Topic: Desenvolvimento, Sustentabilidade e Meio Ambiente

Reviewed anonymously in the process of blind peer.
Received: 06/06/2021

Approved: 28/06/2021
Anderson Saccol Ferreira

Universidade do Oeste de Santa Catarina, Brasil

http://lattes.cnpq.br/4850843904897537

http://orcid.org/0000-0002-6237-9912

anderson.ferreira@unoesc.edu.br

\section{Referencing this:}

FERREIRA, A. S.. Cidades inteligentes e sustentáveis: análise e definições acerca da literatura. Revista Ibero Americana de Ciências Ambientais, v.12, n.6, p.512-521, 2021. DOI: http://doi.org/10.6008/CBPC2179-6858.2021.006.0042 


\section{INTRODUÇÃO}

As cidades contemporâneas enfrentam diversos desafios no existente construído. O meio ambiental já está associado a inúmeros fatores de degradação que estão relacionados ao impacto social, econômico e o uso insustentável de energia e emissão de gases. Isso vem como acelerador para o aumento da poluição do ar, da água, o que torna o uso do solo impróprio para o desenho urbano. Ainda, a ruptura da mobilidade e ineficácia da acessibilidade que gera transtornos no transporte e congestionamento de tráfego, diminuição de segurança e saúde nas cidades.

As cidades ocupam menos de $2 \%$ de massa da terra, os residentes urbanos consomem mais de três quartos dos recursos naturais do mundo e são os principais responsáveis pelas emissões de gases de efeito estufa - GEE (NAM et al., 2011). Esse crescimento desordenado aumenta a variedade de problemas que comprometem a sustentabilidade ambiental, econômica e social das cidades contemporâneas (NEIROTTI et al., 2014). Nesse cenário, a aceleração da urbanização da origem a inúmeros desafios associados a intensidade de consumo, ao congestionamento endêmico, a saturação das redes de transportes a poluição do ar, água, esgotamento dos recursos desigualdade social e vulnerabilidade das pessoas (BIBRI et al., 2017a). Com o agrupamento dinâmico de edificações, infraestrutura, pessoas e dos recursos, a urbanização pressiona os sistemas urbanos estressando a vida urbana em funções e serviços operacionais (BIBRI, 2013).

E dessa forma, vários conceitos vêm surgindo relacionados a qualidade de vida das pessoas nas cidades. Alguns conceitos como cidades jardins, cidades modernas e posteriormente cidades sustentáveis. Atualmente o conceito que vem ganhando força é de cidades inteligentes e sustentáveis.

Esse conceito de Smart Sustainable Cities (SSC), ganha cada vez mais atenção ao redor do globo, principalmente em resposta ao futuro potencial dos desafios ambientais e o aumento de populações que vivem em cidades (NASRAWI et al., 2015). Nesse pensamento Townsend (2013), destaca que o desafio é garantir que as cidades possam oferecer para as gerações atuais e futuras melhores condições de vida aos seus cidadãos. Nam et al. (2011) apontam que o conceito de cidades sustentáveis e inteligentes veio à tona atraindo muitos pesquisadores na busca de formas de promover o desenvolvimento urbano. Mas afinal, o que caracteriza uma cidade inteligente? E qual a relação entre sustentabilidade e inteligência? Quais são os conceitos determinantes para tornar-se uma cidade inteligente e sustentável?

Esse estudo tem como objetivo compreender os conceitos que descrevem as cidades inteligentes e sustentáveis. Adotou como procedimento metodológico a revisão da literatura onde foram analisados 32 estudos científicos. Para a análise foram adotados critérios relacionados ao foco, característica, encadeamento, qualidade, marco teórico e unidade de análise. Após os artigos foram tabulados extraindo os conceitos descritos na literatura.

Como resultados de pesquisa encontramos os seguintes agrupamentos: qualidade de vida, infraestrutura, tecnologia de informação e comunicação-TIC, inteligência, cidadão, sociedade, meio ambiente, sustentabilidade, governança, gestão, economia, finanças e mobilidade. A revisão demonstrou vários conceitos que a literatura utiliza para definir as cidades inteligentes e sustentáveis, e mostra que não 
há um consenso entre as definições, mas que o uso de tecnologias de informação pode auxiliar no desenvolvimento na gestão e na qualidade de vida das cidades. Por fim, o artigo aponta uma breve definição destacando aspectos relacionados à inteligência e sustentabilidade das cidades.

Além dessa introdução o artigo esta estrutura com a revisão teórica que busca nortear a leitura por meio de vários conceitos e definições das cidades inteligentes e sustentáveis. Na sequência os procedimentos metodológicos e os resultados de pesquisa finalizando com as considerações finais do estudo.

\section{REVISÃO TEÓRICA}

\section{Cidades inteligentes e sustentáveis}

No ano de 2007, o mundo observou inúmeras mudanças nas cidades, uma delas foi ver que o número de pessoas vivendo nos espaços urbanos ultrapassou o rural, e a proporção de pessoal poderá ser superior a 70\% até 2050 (FGSSC, 2014). Bibri et al. (2017a) relatam que as cidades contemporâneas consomem aproximadamente $80 \%$ dos recursos, são grandes consumidores energéticos e contribuem significativamente para emissão de gases do efeito estufa (GEE). Fato esse, gerado pelo aumento da concentração populacional urbana e da intensidade da relação ecológica com as atividades econômicas e sociais (BIBRI et al., 2017b). Seus sistemas urbanos, operam e organizam a vida na cidade como a infraestrutura, os serviços, o ecossistema, os serviços humanos e administração e estes estão em crescente pressão devido ao enorme desafio da sustentabilidade, aliado a maior onda de urbanização da história (BIBRI et al., 2017b).

Esse reflexo é decorrente das mudanças na sociedade, as pessoas mudam para as áreas urbanizadas em busca de melhores oportunidades de trabalho. 0 número de pessoas que migram para áreas urbanas leva a questões mais complexas, como o congestionamento de infraestrutura, uso excessivo dos recursos naturais, educação, saneamento e serviços de saúde. Estes problemas são perceptíveis diariamente na vida contemporânea das cidades e obrigam os gestores a criar estratégias para melhorar o bem-estar nos núcleos urbanizados.

Mas o que de fato caracteriza uma cidade inteligente e sustentável, quais são os conceitos determinantes que tornam esses núcleos urbanos inteligentes e sustentáveis. O termo cidades inteligentes não é recente, os primeiros registros apontam meados dos anos de 1800, utilizado para descrever as novas cidades do oeste americano e como elas eram eficientes e autônomas (YIGITCANLAR et al., 2018). Já na contemporaneidade, sua origem emerge na década de 1990 referenciando o modelo de urbanização sustentável (EGER, 2009; ALBINO et al., 2015; SUSANTI et al., 2016).

O conceito de cidades inteligentes desde 1990 até a atualidade evoluiu muito e trouxe para o seu contexto uma base tecnológica de desenvolvimento que opera a gestão da cidade e a implantação de soluções de governança, mobilidade, meio ambiente, tráfego e sociedade (BATTARRA et al., 2016; HARRISON et al., 2011; YIGITCANLAR et al., 2008). Hepworth (1990) aponta que a cidade inteligente emergiu como sucessora das relações dos primeiros municípios de informação e na sequência a cidades digitais (COUCLELIS, 2004). Movidos por uma oferta de tecnologia digital e oportunidade de planejamento urbano digital, nesse 
momento o conceito de "cidades inteligentes e sustentável" passa a ter mais popularidade entre os urbanistas (AINA, 2017; PETTIT et al., 2017; YIGITCANLAR, 2006).

Bibri et al. (2017a), descrevem que a cidade inteligente e sustentável surge a partir de um novo fenômeno tecno-urbano, e em 2010 o termo passou a generalizar (NASRAWI et al., 2015; BIBRI et al., 2017b; HÖJER et al., 2014). Há um viés que aponta que cidades sustentáveis e inteligentes são o pressuposto de inovação digital, e podem gerar ganhos em eficiência operacional e integração de sistemas de infraestrutura urbana que beneficiariam o desenvolvimento econômico, a proteção ambiental e a igualdade social (ALLWINKLE, 2011). A outra convecção, é que elas passam a ser caracterizadas de forma que: a) melhora da capacidade de vida urbana, b) o desenvolvimento eficiente de recursos, c) a economia de baixo carbono, d) o uso de TICs para gerenciar sistemas urbanos complexos (ALUSI et al., 2011).

Elas podem ser um facilitador do uso econômico dos recursos através de melhorias tecnológicas e ambientais, visando o desenvolvimento econômico, da construção de riqueza, do progresso social e a segurança ecológica, mantendo um equilíbrio entre os recursos, meio ambiente, informação, fluxo interno de material e informação, interfluxo de materiais do sistema urbano interno-externo, atendendo às necessidades futuras de uma cidade com base em uma avaliação correta e satisfazendo as necessidades atuais de desenvolvimento urbano (JINGZHU, 2011).

As definições da literatura para as cidades inteligentes e sustentáveis ganham impulsos que atraem a atenção clara do seu caminho de desenvolvimento, florescendo a direção futura. Apesar de muitas considerações associadas às cidades inteligentes e sustentáveis há discrepâncias, que Bibri et al. (2017a) descrevem como uma fraca conexão entre os conceitos de inteligência e sustentabilidade ambiental, e nem sempre as tecnologias são utilizadas de forma a contribuírem com a sustentabilidade. Os autores destacam que as cidades sustentáveis abordam conceitos e princípios que ignoram soluções inteligentes, e as cidades inteligentes concentram-se em tecnologias da informação e comunicação modernas e soluções eficientes deixando de considerar ou ignorar os aspectos de design, ou seja, cidades sustentáveis precisam alavancar sua paisagem informativa e cidades inteligentes, sua paisagem física alinhada com a visão de sustentabilidade (BIBRI et al., 2017a).

Portanto, não há uma definição clara das cidades inteligentes e sustentáveis tão pouco elas estabelecem uma linha base para sustentabilidade, ou definem o desenvolvimento sustentável, mas a definição conceitual acerca delas torna-se crucial para o entendimento do seu propósito, principalmente onde a inteligência deve ser utilizada e como deve ser avaliada, pois seus sistemas contribuem para os objetivos de sustentar desenvolvimento (HÖJER et al., 2014).

\section{METODOLOGIA}

A pesquisa consiste em uma revisão da literatura com o propósito de identificar quais são os conceitos de cidades inteligentes e sustentáveis. Dessa forma, a análise foi da base de dados das revistas Scielo, Science Direct (Elsevier), Scopus, Web of Science. O período dos artigos selecionados foi entre 2011 a 2021, com documentos do tipo, article, review article, article in press, research article. O objetivo dessa 
análise é verificar a produção científica que versa sobre práticas das cidades inteligentes e sustentáveis.

Para a busca, foi utilizado as seguintes palavras-chave: "smart and sustainable cities" or "sustainable urban development" or "TIC and smart cities" or "smart governance" or "smart city technology and efficiency" of "smart city concepts" or "characteristics of smart cities". No Quadro 1, apresento os critérios para seleção dos artigos da pesquisa.

Quadro 1: Critérios de avaliação da qualidade.

\begin{tabular}{|l|l|l|}
\hline Critério & Critério de Inclusão & Critério de Exclusão \\
\hline Foco & $\begin{array}{l}\text { Tratar da temática, encadeamento da sustentabilidade e } \\
\text { inteligência no contexto das cidades inteligentes. }\end{array}$ & $\begin{array}{l}\text { Se referir a temática, encadeamento da } \\
\text { sustentabilidade e inteligência no contexto } \\
\text { urbano de forma genérica. }\end{array}$ \\
\hline Acesso & $\begin{array}{l}\text { Tratar diretamente de características do encadeamento de } \\
\text { sustentabilidade ou inteligência no contexto das cidades, } \\
\text { suas especificidades e maneiras de operacionalizá-las. }\end{array}$ & $\begin{array}{l}\text { Não abordar diretamente caracter da } \\
\text { sustentabilidade e inteligência no contexto } \\
\text { das cidades ou suas especificidades na } \\
\text { organização. }\end{array}$ \\
\hline Qualidade & $\begin{array}{l}\text { Localizar o arquivo do documento na íntegra online e de } \\
\text { forma gratuita. Estar redigido em inglês ou português }\end{array}$ & $\begin{array}{l}\text { Não possuir acesso ao trabalho, não estar } \\
\text { redigido em inglês ou português. }\end{array}$ \\
\hline $\begin{array}{l}\text { Unidade de } \\
\text { análise }\end{array}$ & $\begin{array}{l}\text { Realização do encadeamento: qualidade de vida, } \\
\text { infraestrutura, tecnologia de informação e comunicação-TIC, } \\
\text { cidadão, sociedade, meio ambiente, sustentabilidade, } \\
\text { governança, economia e mobilidade. }\end{array}$ & $\begin{array}{l}\text { Periódico científico sem revisão de pares, } \\
\text { jornais, revistas correntes, conferências, livros } \\
\text { e sites. }\end{array}$ \\
\hline $\begin{array}{l}\text { Tratar de encadeamento de sustentabilidade } \\
\text { ou inteligência em outros contextos } \\
\text { diferentes dos relacionados. }\end{array}$ \\
\hline
\end{tabular}

Na primeira etapa foram selecionados 85 artigos científicos dos quais 53 foram excluídos por não atenderem aos critérios de avaliação da qualidade descritos no quadro 1. No total foram analisados 32 artigos, sendo que a maioria se concentra na plataforma Science Direct (Elsevier). Os estudos selecionados foram tabulados na seguinte ordem: autor, ano, objetivo do estudo, principais variáveis, conceito de cidades inteligentes e sustentáveis usado como diretriz e ou referência no estudo, práticas de cidades inteligentes, benefícios da adoção das cidades inteligentes, contribuição teórica do estudo, limitações da pesquisa e recomendações para futuros estudos.

Após a tabulação dos dados iniciou-se a análise identificando os principais conceitos que descrevem a cidade inteligente e sustentável e registrados no Quadro 2, da mesma maneira, identificar a estrutura dos principais agrupamentos em que as palavras-chave relacionam as cidades inteligentes e sustentáveis.

\section{RESULTADOS E DISCUSSÃO}

Ao analisar a literatura encontramos 335 ocorrências, e, em muitos casos estavam estruturadas nos seguintes agrupamentos: a) qualidade de vida, b) infraestrutura e serviços, c) tecnologia de informação e comunicação-TIC, inteligência, d) cidadão e sociedade, e) meio ambiente e sustentabilidade, f) governança e gestão, g) economia e finanças, h) mobilidade. Os TICs e inteligência lideram com mais de $25 \%$ dos artigos analisados, seguidos de meio ambiente e sustentabilidade com $20 \%$, infraestrutura e serviços com $18 \%$, governança, gestão com $12 \%$, cidadãos e sociedade $5 \%$, economia e finanças $8 \%$, qualidade de vida $6 \%$ e mobilidade com $4 \%$.

Juntos, os oitos motivadores caracterizam uma estrutura de cidade inteligente e sustentável, onde 
cada um deles representa uma dimensão que organiza uma cidade. Essa mesma ótica é compartilhada por Nam et al. (2011), que descrevem uma cidade inteligente como: a) Tecnologia; b) Pessoas; c) Instituições. Ainda, pode ser definida como integração harmoniosa de infraestruturas físicas, tecnologia da informação, sociais, humanas e de negócios, os quais auxiliam para obter uma inteligência coletiva e com uso adequado de toda a informação interconectada disponível, de forma a melhorar o entendimento sobre as operações e otimizar os recursos limitados (HARRISON et al., 2010).

Uma outra linha teórica aponta que as cidades inteligentes poderiam auxiliar na redução dos efeitos de emissão de gases do efeito estufa e uso racional de energias renováveis (MITCHELL, 2000; GESI, 2020). Estas cidades podem melhorar o uso dos recursos públicos, serviços prestados e qualidade de vida, mantendo baixo o custo das operações públicas (ZANELLA et al., 2014), além do transporte dentro das cidades reduzindo os congestionamentos de tráfego (VANOLO, 2013).

Na literatura temos vários conceitos que definem as cidades inteligentes e sustentáveis, e com base nos agrupamentos temos as definições como: a) qualidade de vida, b) TIC, c) infraestrutura; d) pessoas, e) ambiental, f) economia, e, g) governança. Para a classificação das cidades sustentáveis foi utilizado como norteador três aspectos: ambiental, econômico e social. O Quadro 2 aborda está subdividido em autor, tema, classificação e definições encontradas na literatura que conceituam as cidades inteligentes e sustentáveis.

Quadro 2: Definições e conceitos de cidades inteligentes e sustentáveis.

\begin{tabular}{|c|c|c|c|}
\hline Autores & Tema & Classificação & Definições \\
\hline $\begin{array}{l}\text { Nam et al. } \\
\text { (2011). }\end{array}$ & $\begin{array}{ll}\text { Pessoas: } & \text { TIC; } \\
\text { Qualidade de vida; } \\
\text { Governança. }\end{array}$ & $\begin{array}{l}\text { Cidades } \\
\text { inteligentes } \\
\text { Cidades } \\
\text { sustentáveis }\end{array}$ & $\begin{array}{l}\text { Uma cidade é inteligente quando investe em capital humano, social e } \\
\text { infraestrutura de TIC que alimentam o crescimento sustentável e } \\
\text { melhoram a qualidade de vida, por meio da governança participativa }\end{array}$ \\
\hline $\begin{array}{l}\text { Caragliu et al. } \\
\text { (2011). }\end{array}$ & $\begin{array}{l}\text { Pessoas; Governança; } \\
\text { TIC; } \\
\text { Infraestrutura } \\
\text { Ambiental. }\end{array}$ & $\begin{array}{l}\text { Cidades } \\
\text { inteligentes } \\
\text { Cidades } \\
\text { sustentáveis }\end{array}$ & $\begin{array}{l}\text { As cidades inteligentes oferecem um modo de governança em qual } \\
\text { equidade social e proteção ambiental podem ser alcançadas em } \\
\text { paralelo com o crescimento econômico catalisado digitalmente. A } \\
\text { cidade inteligente com sua mídia digital, eficiente e infraestrutura } \\
\text { integrada é posicionada como um facilitador de desenvolvimento } \\
\text { sustentável, alinhando os objetivos do meio ambiente à proteção, } \\
\text { equidade social e desenvolvimento econômico. }\end{array}$ \\
\hline $\begin{array}{l}\text { Lee et al. } \\
(2014) \text {. }\end{array}$ & TIC; Economia. & $\begin{array}{l}\text { Cidades } \\
\text { inteligentes } \\
\text { Cidades } \\
\text { sustentáveis }\end{array}$ & $\begin{array}{l}\text { A cidade inteligente e sustentável pode ser obtida por meio da adoção } \\
\text { de um sistema público-privado parceiro e eficaz, conformando } \\
\text { modelos de negócios de valor agregado e incorporando tecnologias } \\
\text { emergentes em sistemas de cidades inteligentes. }\end{array}$ \\
\hline $\begin{array}{l}\text { Lee et al. } \\
(2014) \text {. }\end{array}$ & $\begin{array}{l}\text { Pessoas; } \\
\text { Governança; } \\
\text { Infraestrutura }\end{array}$ & $\begin{array}{l}\text { Cidades } \\
\text { inteligentes }\end{array}$ & $\begin{array}{l}\text { a) inovação de serviço; b) formação de parcerias; c) urbanização pro- } \\
\text { atuação efetiva; d) integração da infraestrutura da cidade inteligente; } \\
\text { e) cidade inteligente e governança. }\end{array}$ \\
\hline $\begin{array}{l}\text { Angelidou } \\
\text { (2015). }\end{array}$ & Economia; TICs & $\begin{array}{l}\text { Cidades } \\
\text { inteligentes }\end{array}$ & $\begin{array}{l}\text { Uma cidade inteligente com base em quatro forças principais, a saber: } \\
\text { a) futuros urbanos; b) conhecimento; c) economia e inovação; d) } \\
\text { impulso de tecnologia; }\end{array}$ \\
\hline $\begin{array}{l}\text { Jong et al. } \\
(2015)\end{array}$ & $\begin{array}{l}\text { Governança; } \\
\text { Infraestrutura; } \\
\text { TIC; }\end{array}$ & $\begin{array}{l}\text { Cidades } \\
\text { inteligentes }\end{array}$ & $\begin{array}{l}\text { A criação de cidades inteligentes e a garantia de sua sustentabilidade } \\
\text { desde o início, o que pode ajudar a resolver os problemas de } \\
\text { urbanização e levar as cidades ao desenvolvimento sustentável. }\end{array}$ \\
\hline $\begin{array}{l}\text { Joshi et al. } \\
(2016) \text {. }\end{array}$ & $\begin{array}{l}\text { Pessoas; Governança; } \\
\text { TIC; }\end{array}$ & $\begin{array}{l}\text { Cidades } \\
\text { sustentáveis }\end{array}$ & $\begin{array}{l}\text { Identificou seis pilares importantes para o desenvolvimento de uma } \\
\text { estrutura de cidade inteligente: a) social; b) gestão; c) econômico; d) } \\
\text { legal; e) tecnologia; f) sustentabilidade. }\end{array}$ \\
\hline March (2016). & Economia. & $\begin{array}{l}\text { Cidades } \\
\text { sustentáveis }\end{array}$ & $\begin{array}{l}\text { O desenvolvimento sustentável poderia ser melhor facilitado pelo } \\
\text { imperativo de desenvolvimento de cidade inteligente a partir do } \\
\text { crescimento econômico para decrescer. Essa mudança seria o } \\
\text { prenúncio do surgimento de economias urbanas pós-capitalistas e } \\
\text { mediadas digitalmente, onde o código aberto e a fabricação digital } \\
\text { desempenham um papel central na economia da cidade e na vida de } \\
\text { seus residentes. }\end{array}$ \\
\hline
\end{tabular}




\begin{tabular}{|c|c|c|c|}
\hline $\begin{array}{l}\text { Meijer et al. } \\
(2016)\end{array}$ & $\begin{array}{l}\text { Pessoas; TIC } \\
\text { Governança. }\end{array}$ & $\begin{array}{l}\text { Cidades } \\
\text { inteligentes }\end{array}$ & $\begin{array}{l}\text { A inteligente da cidade deve estar relacionada com pessoas } \\
\text { inteligentes (com foco em recursos humanos); colaborar (governança) } \\
\text { e técnicas (com tecnologia). }\end{array}$ \\
\hline $\begin{array}{l}\text { Ahvenniemi } \\
\text { et al. (2017) }\end{array}$ & $\begin{array}{l}\text { TIC, Qualidade de vida; } \\
\text { ambiental; }\end{array}$ & $\begin{array}{l}\text { Cidades } \\
\text { inteligentes }\end{array}$ & $\begin{array}{l}\text { As Cidades inteligentes não possuem um significado claro e } \\
\text { consistente, mas há indicações de que o uso de diversas tecnologias } \\
\text { modernas possibilita alcançar melhor qualidade de vida e diminuição } \\
\text { do impacto ambiental. }\end{array}$ \\
\hline $\begin{array}{l}\text { Fernandez- } \\
\text { Anez et al. } \\
(2018)\end{array}$ & Governança; & $\begin{array}{l}\text { Cidades } \\
\text { inteligentes }\end{array}$ & $\begin{array}{l}\text { Cidade inteligente como um sistema integrado e multidimensional, e } \\
\text { atenta ligar três questões principais: a) o papel-chave da governança } \\
\text { e envolvimento das partes interessadas; b) a importância de exibir } \\
\text { uma visão compreensiva de projetos e dimensões de cidades } \\
\text { inteligentes; c) a compreensão da cidade inteligente como uma } \\
\text { ferramenta para enfrentar os desafios urbanos. }\end{array}$ \\
\hline $\begin{array}{l}\text { Yigitcanlar et } \\
\text { al. (2018). }\end{array}$ & $\begin{array}{l}\text { Pessoas; } \quad \text { TIC; } \\
\text { Governança; } \\
\text { Economia; }\end{array}$ & $\begin{array}{l}\text { Cidades } \\
\text { inteligentes }\end{array}$ & $\begin{array}{l}\text { Descrevem três motivadores para cidades inteligentes: comunidade, } \\
\text { tecnologia e política. Esses que estão ligados a seis resultados } \\
\text { desejados: a) produtividade, b) sustentabilidade, c) acessibilidade, d) } \\
\text { bem-estar, e) habitabilidade, f) governança. Juntos montam uma } \\
\text { estrutura de cidade inteligente, onde cada um deles representa uma } \\
\text { dimensão distinta da noção de cidades inteligentes. }\end{array}$ \\
\hline $\begin{array}{l}\text { Martin et al. } \\
\text { (2018). }\end{array}$ & $\begin{array}{l}\text { Ambiental; Pessoas; } \\
\text { Qualidade de vida; }\end{array}$ & $\begin{array}{l}\text { Cidades } \\
\text { inteligentes }\end{array}$ & $\begin{array}{l}\text { É importante projetar projetos e programas de cidades inteligentes de } \\
\text { maneiras que abordam questões ambientais e sociais amplas. Pode } \\
\text { ser feito envolvendo uma gama maior de partes interessadas na } \\
\text { concepção de projetos e através da extensão do monitoramento e } \\
\text { avaliação para incluir medidas mais amplas de social e desempenho } \\
\text { ambiental. }\end{array}$ \\
\hline $\begin{array}{l}\text { Ismagilova et } \\
\text { al. (2019) }\end{array}$ & $\begin{array}{l}\text { TIC, Qualidade de } \\
\text { Vida; Ambiental; } \\
\text { Governança. }\end{array}$ & $\begin{array}{l}\text { Cidades } \\
\text { inteligentes } \\
\text { Cidades } \\
\text { sustentáveis }\end{array}$ & $\begin{array}{l}\text { As cidades inteligentes possuem uma abordagem centrada em } \\
\text { sistema de informação, para uso inteligente de interação, } \\
\text { sustentabilidade, cidadania e qualidade de vida, dentro de uma } \\
\text { infraestrutura interativa para fornecer serviços avançados e } \\
\text { inovadores aos cidadãos, impactando na qualidade de vida e na gestão } \\
\text { sustentável de recursos naturais. }\end{array}$ \\
\hline $\begin{array}{l}\text { Antwi-Afari et } \\
\text { al. (2021) }\end{array}$ & $\begin{array}{l}\text { Pessoas; Qualidade de } \\
\text { vida; infraestrutura; } \\
\text { Ambiental; } \\
\text { Governança; } \\
\text { Economia }\end{array}$ & $\begin{array}{l}\text { Cidades } \\
\text { inteligentes } \\
\text { Cidades } \\
\text { sustentáveis }\end{array}$ & $\begin{array}{l}\text { Adotam seis dimensões para conceituar a estrutura urbana das } \\
\text { cidades inteligentes: a) vida; b) pessoas; c) mobilidade; d) economia, } \\
\text { e) governança; f) meio ambiente. }\end{array}$ \\
\hline $\begin{array}{l}\text { Csukás et al. } \\
\text { (2021) }\end{array}$ & $\begin{array}{l}\text { Ambiental; TIC } \\
\text { Pessoas; Governança; }\end{array}$ & $\begin{array}{l}\text { Cidades } \\
\text { inteligentes }\end{array}$ & $\begin{array}{l}\text { Elencam quatro modelos de cidades inteligentes sendo: a) A cidade } \\
\text { verde, onde se concentrando em objetivos relacionados ao meio } \\
\text { ambiente; b) A App City se concentrando no desenvolvimento e } \\
\text { implantação de plataformas e aplicativos de TICs para fornecer } \\
\text { qualidade de vida e melhorias diretamente para os cidadãos; c) A } \\
\text { cidade socialmente sensível; d) A cidade participativa, engajamento } \\
\text { cidadão como em foco. }\end{array}$ \\
\hline
\end{tabular}

A grande maioria dos textos apontam um viés para cidades inteligentes, principalmente para uma relação com TICs e qualidade de vida, TICs e pessoas, TICs e governança e os TICs com a sustentabilidade. Vários fatores que designam sustentabilidade, como meio ambiental, econômico, político institucional estão presentes nas cidades inteligentes, de forma a serem norteados pelas tecnologias da informação e comunicação.

A uma relação que, inclusive, a inteligência das cidades poderia melhor o progresso em áreas como produção de energia, mobilidade e transporte, TICs, e criar várias oportunidades interdisciplinares para melhorar os serviços e, ao mesmo tempo, reduzir a energia e emissões de gases de efeito estufa (GEE) (FLETCHER et al., 2007; CHAIGNEAU et al., 2016). Dessa forma, as cidades inteligentes podem ser vistas como uma solução para o desenvolvimento urbano sustentável e as conturbações que levam a uma urbanização aprimorada.

Com base na análise pode-se definir que uma cidade sustentável e inteligente é aquela que aproveita os sistemas de tecnologias da informação e comunicação em uma modelo adaptável, confiável, escalável e 
seguro a fim de melhorar a qualidade de vida, infraestrutura, os serviços, o meio ambiente, governança e a gestão. Ainda garante o crescimento econômico de forma tangível oferecendo padrões de vida mais elevados com oportunidade de emprego e renda para as pessoas, sem esquecer de melhorar o bem-estar, a saúde, a segurança e a educação. A cidade inteligente e sustentável atende as necessidades de hoje sem sacrificar as necessidades das próximas gerações garantindo o meio ambiental, econômico e social.

Assim pode-se caracterizar a cidade inteligente e sustentável como: inovadora, adaptável e segura, usa a tecnologia de informação e comunicação (TICs) para melhorar a qualidade de vida, elevar as oportunidades de trabalho e seu sistema de governança local, é caracterizado pelo profundo envolvimento e inclusão dos cidadãos, de forma garantir as necessidades das presentes e futuras gerações respeitando os aspectos sociais, econômicas e ambientais. A junção de vários aspectos monta uma estrutura de cidade inteligente e sustentável, onde cada um deles representa uma dimensão distinta da noção que caracteriza e conceitua as cidades inteligentes e sustentáveis.

\section{CONCLUSÕES}

O artigo fornece uma visão abrangente das cidades inteligentes e sustentáveis e busca caracterizar quais são os conceitos determinantes dessas cidades. Por meio da revisão da literatura buscou compreender os principais conceitos que descrevem as cidades inteligentes e sustentáveis.

Os resultados dessa revisão da literatura permitem estabelecer uma visão panorâmica dos conceitos descritos na literatura acerca do desenvolvimento das cidades inteligentes e sustentáveis. Os sistemas de tecnologias da informação e comunicação - TICs estão presentes na maior parte da literatura como forma de auxiliar a inovação e tornar a cidade adaptável e segura. A revisão aponta que as cidades inteligentes e sustentáveis possuem características semelhantes visando a qualidade de vida, infraestrutura, pessoas, sistemas de informação, governança e meio ambiente.

As constatações do estudo são significativas para o planejamento e desenvolvimento urbano das cidades e estabelecem motivadores que caracterizam uma estrutura de cidade inteligente e sustentável, de forma que cada um deles representa uma dimensão que organiza as cidades.

Os futuros estudos poderiam investigar as vantagens e discrepâncias entre os conceitos das cidades inteligentes e sustentáveis, assim como mensurar os elementos que caracterizam estas cidades. Ainda, pode ser investigado a relação dos TICs como ferramenta de integração entre inteligência e sustentabilidade, e se esta relação ocorre na prática, e como ela interage entres os modelos de cidades sustentáveis como a ecocidade, cidade compacta, cidade verde. Este estudo limita-se a uma análise da revisão teórica da literatura que se baseia em modelos e conceitos teóricos de cidades inteligentes e sustentáveis.

\section{REFERÊNCIAS}

AHVENNI EMI, H.; HUOVILA, A.; PINTO-SEPPÄ, I.; AIRAKSINEN, M.. What are the differences between sustainable and smart cities? Cities, v.60, p.234-245, 2017. DOI:_https://doi.org/10.1016/i.cities.2016.09.009
AINA, Y. A.. Achieving smart sustainable cities with GeolCT support: The Saudi evolving smart cities. Cities, v.71, p.4958, 2017. DOI: https://doi.org/10.1016/j.cities.2017.07.007 
ALBINO, V.; BERARDI, U.; DANGELICO, R. M.. Smart cities: Definitions, dimensions, performance, and initiatives. Journal of Urban Technology, v.22, n.1, p.3-21, 2015. DOI: http://dx.doi.org/10.1080/10630732.2014.942092

ALUSI, A.; ECCLES, R. G.; EDMONDSON, A. C.; ZUZUL, T.. Sustainable Cities: Oxymoron or the Shape of the Future. Havard Business School, 2011.

ANGELIDOU, M.. Smart cities: A conjuncture of four forces. Cities, v.47, p.95-106, 2015. DOI:

https://doi.org/10.1016/j.cities.2015.05.004

ANTWI-AFARI, P.; GRAFT, O.; SIMONS, B.; DEBRAH, C.; GHANSAH, F.. Sustainability guidelines to attaining smart sustainable cities in developing countries: A Ghanaian context. Sustainable Futures, v.3, 2021. DOI: https://doi.org/10.1016/i.sftr.2021.100044

BATTARRA, R.; GARGIULO, C.; PAPPALARDO, G.; BOIANO, D. A.; OLIVA, J. S.. Planning in the era of information and communication Technologies - Discussing the "label: smart" in South-European cities with environmental and socioeconomic challenges. Cities, v.59, p.1-7, 2016. DOI: https://doi.org/10.1016/j.cities.2016.05.007

BIBRI, S. E.. ICT for sustainable urban development in the European Information Society: A discursive investigation of energy efficiency technology. Thesis (Master) - School of Culture and Society, Malmö University, Malmö, 2013.

BIBRI, S. E.; KROGSTIE, J.. Smart sustainable cities of the future: An extensive interdisciplinary literature review. Sustainable Cities and Society, v.31, p.183-212, 2017a. DOI: http://dx.doi.org/10.1016/j.scs.2017.02.016

BIBRI, S. E.; KROGSTIE, J.. On the social shaping dimensions of smart sustainable cities: A study in science, technology, and society. Sustainable Cities and Society, v.29, p.219-246, 2017b. DOI: 10.1016/j.scs.2016.11.004

CARAGLIU, A.; DEL BO, C.; NIJKAMP, P.. Smart cities in Europe. J. Urban Technol., v.18, p.65-82, 2011. DOI: http://dx.doi.org/10.1080/10630732.2011.601117

CHAIGNEAU, T.; BROWN, K.. Challenging the win-win discourse on conservation and development: analysing support for marine protected areas, Ecology and Society, v.21, n.1, 2016. DOI: http://dx.doi.org/10.5751/ES-08204$\underline{210136}$

COUCLELIS, H.. The construction of the digital city. Environment and Planning B: Urban Analytics and City Science, n.31, p.5-19, 2004. DOI: https://doi.org/10.1068/b1299

EGER, J. M.. Smart growth, smart cities, and the crisis at the pump a worldwide phenomenon. The Journal of $E$ -

Government Policy and Regulation, n.32, v.1, p.47-53, 2009. DOI: http://doi.org/10.3233/IWA-2009-0164

FERNANDEZ-ANEZ, V., FERNÁNDEZ-GÜELL, J. M.; GIFFINGER, R.. Smart City implementation and discourses: An integrated conceptual model. The case of Vienna. Cities, 78, p.4-16, 2018. DOI: https://doi.org/10.1016/i.cities.2017.12.004
FLETCHER, S.; POTTS, J.. Ocean citizenship: an emergent geographical concept, Coastal Management., v.35, n.4, p.511-524, 2007. DOI:

https://doi.org/10.1080/08920750701525818

FGSSC. Focus Group on Smart Sustainable Cities. Smart sustainable cities: An analysis of definitions.

Telecommunication Standardization Sector Of ITU, 2014

GESI, S.. Enabling the Low Carbon Economy in the Information Age, London, 2020.

HARRISON, C.; ECKMAN, B.; HAMILTON, R.; HARTSWICK, P.; KALAGNANAM, J.; PARASZCZAK, J.; WILLIAMS, P.. Foundations for smarter cities, IBM Journal of Research and Development, v.54, n.4, p.1-16, 2010. DOI: http://doi.org/10.1147/JRD.2010.2048257

HARRISON, C.; DONNELLY, I. A.. A theory of smart cities. Proceedings of the 55th Annual Meeting of the ISSS, Hull, v.55, n.1, p.1-15, 2011.

HEPWORTH, M. E.. Planning for the information city: the challenge and response. Urban Stud., n.27, p.537-558, 1990. DOI: http://dx.doi.org/10.1080/00420989020080501

HÖJER, M.; WANGEL, S.. Smart sustainable cities: Definition and challenges. In: HILTY, L.; AEBISCHER, B.. ICT innovations for sustainability. Berlin: Springer-verlag, 2014. p.333-349. DOI: http://doi.org/10.1007/978-3-319-09228-7 20

ISMAGILOVA, E.; HUGHES, L.; YOGESH, K.; DWIVEDI, K.; RAMAN, R.. Smart cities: advances in research: an information systems perspective. International Journal of Information Management, v.47, p.88-100, 2019. DOI: https://doi.org/10.1016/j.ijinfomgt.2019.01.004

JINGZHU, Z.. Towards Sustainable Cities in China, Springer Briefs in Environmental Science. Springer, 2011.

JONG, M.; JOSS, S.; SCHRAVEN, D.; ZHAN, C.; WEIJNEN, M. Sustainable smart-resilient-low carbon-eco-knowledge cities; making sense of a multitude of concepts promoting sustainable urbanization, Journal of Cleaner Production, v.109, p.25-38, 2015. DOI:

https://doi.org/10.1016/j.jclepro.2015.02.004

JOSHI, S.; SAXENA, S.; GODBOLE, T.. Developing smart cities: An integrated framework. Procedia Computer Science, v.93, p.902-909, 2016. DOI:

https://doi.org/10.1016/j.procs.2016.07.258

LEE, J. H.; HANCOCK, M. G.; HU, M. C.. Towards an effective framework for building smart cities: Lessons from Seoul and San Francisco. Technological Forecasting and Social Change, v.89, p.80-99, 2014. DOI:

https://doi.org/10.1016/i.techfore.2013.08.033

MARCH, H.. The Smart City and other ICT-led technoimaginaries: any room for dialogue with degrowth? Journal of Cleaner Production, v.197, p.1694-1703, 2016. DOI: https://doi.org/10.1016/j.jclepro.2016.09.154

MARTIN, C. J.; EVANS, J.; KARVONEN, A.. Smart and sustainable? Five tensions in the visions and practices of the smart-sustainable city in Europe and North America.

Technological Forecasting and Social Change, v.133, p.269- 
278, 2018. DOI:

https://doi.org/10.1016/i.techfore.2018.01.005

MEIJER, A.; BOLÍVAR, M. P. R.. Governing the smart city: a review of the literature on smart urban governance, International Review of Administrative Sciences, n.82, v, 2, p.392-408, 2016. DOI:

https://doi.org/10.1177/0020852314564308

MITCHELL, W. J.. E-topia. Urban Life, Jim e but Not as We Know it. The MIT Press, Cambridge Mass, 2000. DOI: https://doi.org/10.7551/mitpress/2844.001.0001

NAM, T.; PARDO, T.. Smart city as urban innovation. INTERNATIONAL CONFERENCE ON THEORY AND PRACTICE OF ELECTRONIC GOVERNANCE - ICEGOV'11, 5. Annals. 2011. DOI: https://doi.org/10.1145/2072069.2072100

NAM, T.; PARDO, T.. Conceptualizing Smart City with Dimensions of Technology, People, and Institutions. In: ANNUAL INTERNATIONAL DIGITAL GOVERNMENT RESEARCH CONFERENCE, 12. Annals. 2011. p.282-291. DOI: https://doi.org/10.1145/2037556.2037602

NASRAWI, S.; ADAMS, C.; El-ZAART, A.. A conceptual multidimensional model for assessing smart sustainable cities. JISTEM - Journal of Information Systems and Technology Management, v.12, n.3, 2015. DOI: https://doi.org/10.4301/S1807-17752015000300003

NEIROTTI, P.; MARCO, A.; CAGLIANO, A. C.; MANGANO, G.; SCORRANO, F.. Current trends in smart city initiatives-some stylized facts. Cities, v.38, p.25-36, 2014. DOI: https://doi.org/10.1016/j.cities.2013.12.010

PETTIT, C.; BAKELMUN, A.; LIESKE, S. N.; GLACKIN, S.; THOMSON, G.; SHEARER, H.; NEWMAN, P.. Planning support systems for smart cities. City, Culture and Society, p.1-12, 2017. DOI: http://dx.doi.org/10.1016/i.ccs.2017.10.002

SUSANTI, R.; SOETOMO, S.; BUCHORI, I.; BROTOSUNARYO, P. $M$.. Smart growth, smart city and density: In search of the appropriate indicator for residential density in Indonesia. Procedia - Social and Behavioral Sciences, v.227, 194-201, 2016. DOI: https://doi.org/10.1016/j.sbspro.2016.06.062

TOWNSEND, A.. Smart Cities-Big Data, Civic Hackers and the Quest for a new Utopia. New York: Norton \& Company, 2013.

VANOLO, A.. Smart mentality: the smart city as disciplinary strategy, Urban Studies, v.51, n.5, p.883-898, 2013. DOI: https://doi.org/10.1177/0042098013494427

YIGITCANLAR, T.. Australian local governments' practice and prospects with online planning. Urisa Journal, n.18, v.2, p.717,2006

YIGITCANLAR, T.; FABIAN, L.; COIACETTO, E.. Challenges to urban transport sustainability and smart transport in a tourist city: The Gold Coast, Australia. Open Transportation Journal, n.1, p.19-36, 2008. DOI: http://doi.org/10.2174/1874447800802010029

YIGITCANLAR, T.; KAMRUZZAMAN, M.; BUYS, L.; IOPPOLO, G. I.; SABATINIMARQUES, J.; COSTA, E. M.; YUN, J. J.. Understanding 'smart cities': Intertwining development drivers with desired outcomes in a multidimensional framework. Cities, v.81, p.145-160, 2018. DOI: https://doi.org/10.1016/j.cities.2018.04.003

ZANELLA, A.; BUI, N.; CASTELLANI, A.; VANGELISTA, L.; ZORZI, $M .$. Internet of things for smart cities. IEEE Internet of Things Journal, v.1, n.1, p.22-32, 2014. DOI: http://doi.org/10.1109/JIOT.2014.2306328

A CBPC - Companhia Brasileira de Produção Científica (CNPJ: 11.221.422/0001-03) detém os direitos materiais desta publicação. Os direitos referem-se à publicação do trabalho em qualquer parte do mundo, incluindo os direitos às renovações, expansões e disseminações da contribuição, bem como outros direitos subsidiários. Todos os trabalhos publicados eletronicamente poderão posteriormente ser publicados em coletâneas impressas sob coordenação da Sustenere Publishing, da Companhia Brasileira de Produção Científica e seus parceiros autorizados. Os (as) autores (as) preservam os direitos autorais, mas não têm permissão para a publicação da contribuição em outro meio, impresso ou digital, em português ou em tradução. 